\title{
Research on Hot Spot Temperature Calculation and Analysis of Online Monitoring Method of Oil-immersed Power Transformer Winding
}

\author{
Yun Zhanga, * \\ Department of Electronic and Communication Engineering North China Electric Power \\ University1Baoding 071003, Hebei, China \\ a1034044638@qq.com
}

Keywords: Oil-immersed power transformer, Hot spot temperature, On-line monitoring, MATLAB

\begin{abstract}
Transformer hot spot temperature is the most important reason for affecting the insulation state of the winding. Hot spot position is also one of the most serious areas in transformer oil paper insulation aging. Therefore, to monitor the hot point temperature is of great significance. This paper introduces the indirect calculation model of hot spot temperature of oil-immersed power transformer and the real-time on-line monitoring system. Subsequently the simulation of transformer hot spot temperature instance is enumerated with the MATLAB environment.
\end{abstract}

\section{Introduction}

Power transformers are one of the most important and expensive electrical equipment of power systems, and their stability and safety are directly related to power supply quality and grid stability. At present, the operating system of the transmission system has been properly increased, and the transformer as a transmission system bottleneck, is a key link. Oil-immersed power transformer insulation often uses oil-paper insulation structure type. In this case temperature is the main factor to promote the aging ${ }^{[1]}$. Hot spot is not only the most serious areas where insulation state is affected by temperature, but oil and paper insulation aging are one of the fastest ${ }^{[2]}$. As a result, the transformer insulation performance, life is related to the temperature ${ }^{[3]}$.

However, the current transformer operating limit is based on the average temperature to calculate, while the warmest temperature of the transformer winding and the average temperature difference vary between the transformer structure and cooling methods. Such as utilizing the average temperature to calculate the operating limit, on the one hand it may cause the internal winding overheating and shorten the life of the transformer; the other hand there is waste caused by insufficient load. Therefore, correctly grasp the real-time transformer winding hot spot temperature, can not only ensure transformer safe and healthy operation, but also fully exploit the potential of the equipment, which is beneficial to solve the peak power grid or failure of short-term power supply. As a consequence, monitoring the hot spot temperature of the transformer can provide effective technical support for transformer operation and management.

\section{Measurement Method of Transformer Winding Temperature}

Due to the particularity of the transformer structure, the measurement of the hottest temperature of the transformer winding has long plagued the electric worker. At present the most effective way to determine the hottest temperature of the transformer winding is still an indirect calculation method. To get hot spots, related operators need manual calculation base on Exponential equation solution introduced by IEC 60076-7:2005 "Loading guide for oil-immersed power transformers" ${ }^{4]}$ (referred to as guidelines). 


$$
\begin{aligned}
\theta_{h}(t)= & \theta_{a}+\Delta \theta_{o i}+\left\{\Delta \theta_{o r} \times\left[\frac{1+R \times K^{2}}{1+R}\right]^{x}-\Delta \theta_{o i}\right\} \times f_{1}(t) \\
& +\Delta \theta_{h i}+\left(H g_{r} K^{y}-\Delta \theta_{h i}\right) \times f_{2}(t)
\end{aligned}
$$

The above model applies to loads that vary by step function. For arbitrarily time-varying load coefficient $\mathrm{K}$ and time-varying ambient temperature, it is clear that the differential equation model introduced in the load guide is more reflective of the actual situation. The following focuses on this method.

According to the contents of the load guide, adding the temperature difference between the hot spot temperature in the transformer tank and the temperature of the top oil, the temperature rise of the top oil in the tank and the ambient temperature, the sum is equal to the hot spot temperature.

The differential equation for the temperature of the top oil in the transformer tank is shown below

$$
\left[\frac{1+K^{2} R}{1+R}\right]^{x} \times\left(\Delta \theta_{o r}\right)=k_{11} \tau_{o} \times \frac{d \theta_{o}}{d t}+\left[\theta_{o}-\theta_{a}\right]
$$

The hot-temperature rise equation is the sum of two differential equations

$$
\Delta \theta_{h}=\Delta \theta_{h 1}-\Delta \theta_{h 2}
$$

The two equations are

$$
\begin{aligned}
& k_{21} \times K^{y} \times\left(\Delta \theta_{h r}\right)=k_{22} \times \tau_{w} \times \frac{d \Delta \theta_{h 1}}{d t}+\Delta \theta_{h 1} \\
& \left(k_{21}-1\right) \times K^{y} \times\left(\Delta \theta_{h r}\right)=\left(\frac{\tau_{o}}{k_{22}}\right) \times \frac{d \Delta \theta_{h 2}}{d t}+\Delta \theta_{h 2}
\end{aligned}
$$

The solution of the above equation is then substituted into equation (2) and $\Delta \theta_{n}$ is obtained. The final equation for the hotspot temperature is

$\theta_{n}=\theta_{0}+\Delta \theta_{h}$

Where $K$ represents the load factor; $R$ means the ratio of the load loss to the no-load loss at the rated current; $\theta_{h}$ represents the hot spot temperature $\left({ }^{\circ} \mathrm{C}\right)$; $\theta_{0}$ represents the temperature of the top oil in the tank under the considered load $\left({ }^{\circ} \mathrm{C}\right)$; $\theta_{a}$ is environment temperature; $\Delta \theta_{o r}$ represents the steady-state temperature rise of the top oil in the tank under the rated loss $(K)$;

$\Delta \theta_{\mathrm{hr}}$ is the gradient of the hot spot temperature of the winding under the load under consideration, whose unit is $K ; x$ is called the oil index; $y$ is called the winding index; $k_{11}, k_{21}, k_{22}$ are the thermal model constants; $t$ is the time variable whose unit is min.

Actually it would be cumbersome to use the manual calculation of the model. IEC 60076-7:2005 "Loading guide for oil-immersed power transformers" has an idea of solving the equation-transform the differential equation into a differential equation and then solve the difference equation, which is is relatively simple to operate.

\section{MATLAB Simulation Example}

Assuming that the object under consideration is the information of the hot spot temperature and the life loss sent by an on-line monitoring device, the parameter settings of the transformer and the setting of the input data are as follows (the parameters used are selected when ambient temperature is $30{ }^{\circ} \mathrm{C}$ and the rated hot spot temperature is $110^{\circ} \mathrm{C}$ ). 
Table 1. Transformer parameters in the instance

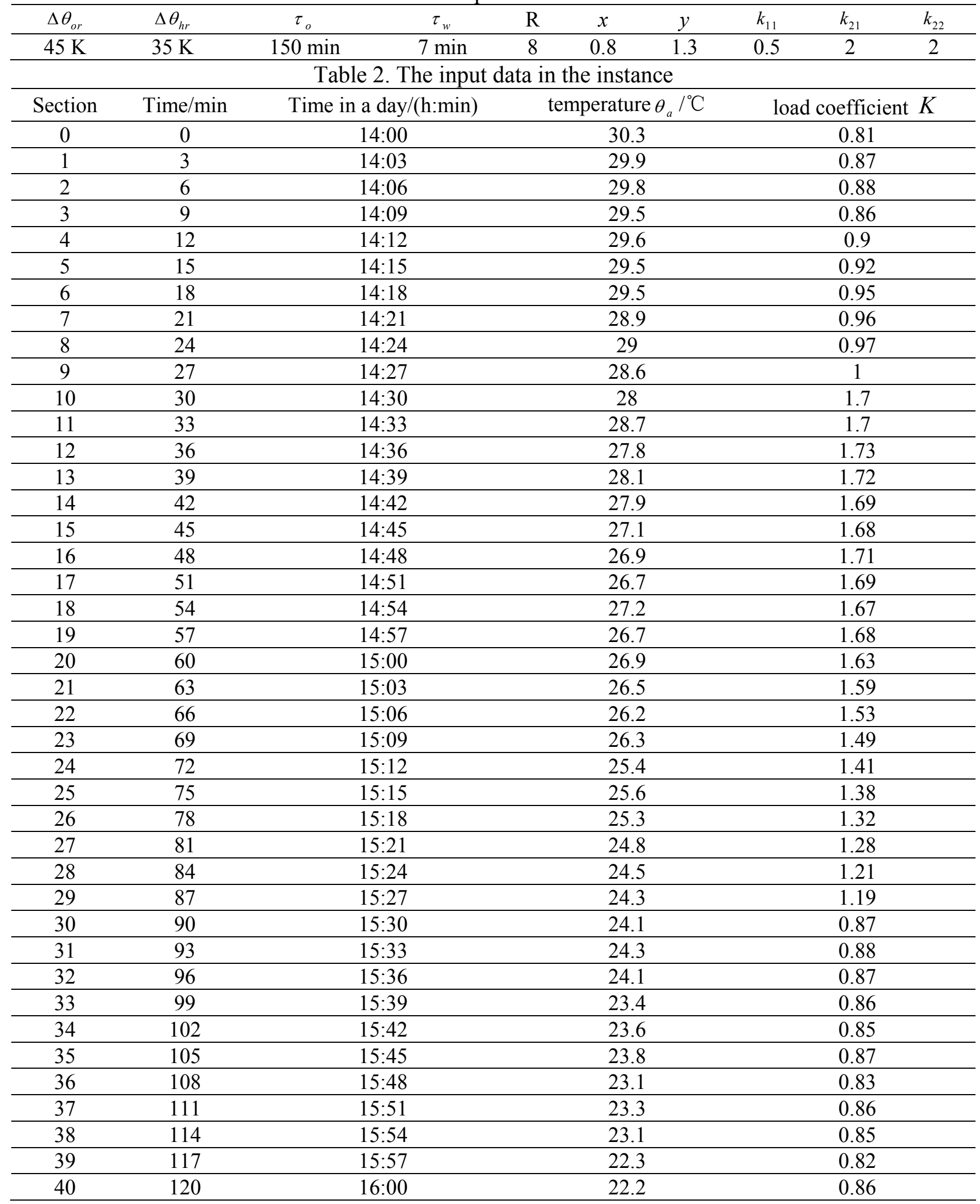

By observing the above table, it can be found that the change of the load does not accord with the characteristics of the step function, so the method used in this simulation example is the differential equation solution introduced in the guideline. Through the operation of the program, the hot spot temperature of oil-immersed power transformer and the top of the oil temperature changes with time. The simulation results are shown in Figure 1. 


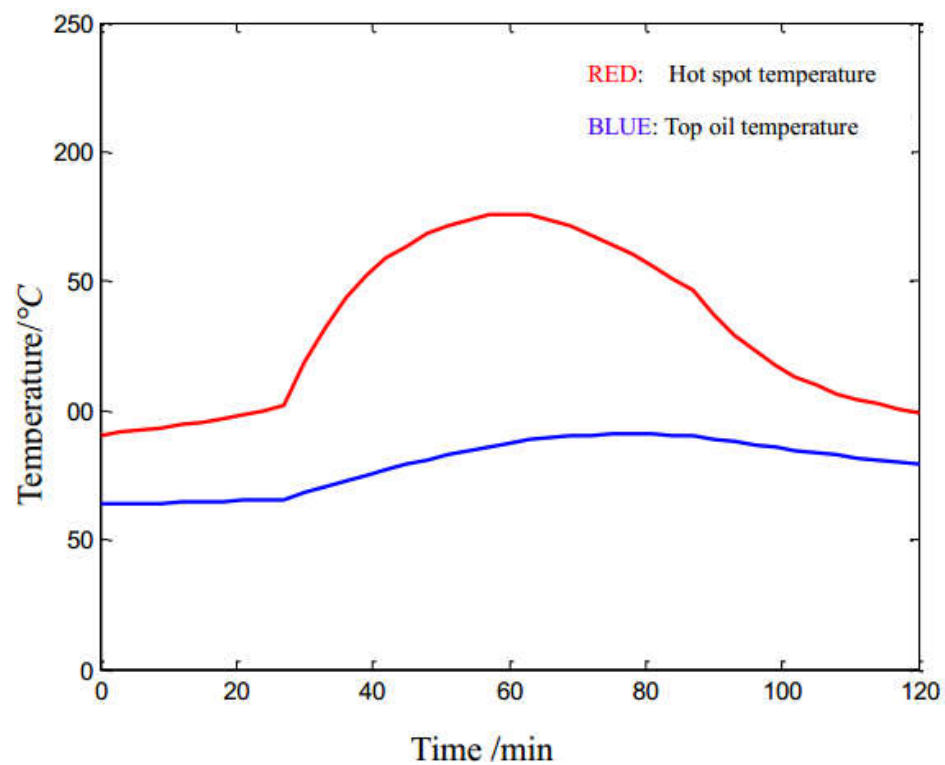

Figure 1. Hot - spot Temperature Measurement Model of Oil - immersed Power Transformer (Based on IEC standards)

\section{Conclusion and Discussion}

In this paper based on the differential equation method in the load guideline, the differential equation is transformed into the difference equation, and then the difference equation is solved to obtain the hot spot temperature. Although this method is simplified, at the same time convert a continuous model into a discrete model, which cannot accurately reflect the reality. In this way it will inevitably lead to increased computing error, while complex calculations often lead low work efficiency.

In view of the above problems, the project can be resolved by the following methods:

(1) Using the industry's latest oil temperature formula to replace the original top of the oil temperature calculation formula. By analyzing the heat generation process inside the transformer, the physical meaning of each parameter in the recommended method of the load guide is explained in detail. On the basis of this, the calculation of the internal heat generation and cooling process of the transformer is carried out to improve the calculation model of the top oil temperature and the calculation part of hot spot temperature.

(2) The differential equation for the hot-point temperature calculation of the transformer winding is solved by a relatively advanced variable-step-length Runge-Kutta method to improve the accuracy of the calculation

\section{Reference}

[1]. Atabak Najafi, Ires Iskender, An improved thermal model for distribution transformer under unbalanced voltage conditions, International Journal of Thermal Sciences, Volume 104, 2016, Pages 373-385, ISSN 1290-0729

[2]. B.T. Desai, H.O. Gupta, M.K. Vasantha, Microprocessor-based on-line monitoring of hot-spot temperature, total consumed life and consumed/saved life in a day for load limit control of power transformer, Journal of Microcomputer Applications, Volume 12, Issue 1, 1989, Pages 41-51, ISSN 0745-7138

[3]. K.T. Muthanna, A. Sarkar, K. Das, K. Waldner, Transformer insulation life assessment, IEEE Trans. Power Deliv. 21 (2006) 150-156.

[4]. Transformers-Part P. 7: loading guide for oil-immersed power transformers [J]. IEC standard, 2005: 60076-7. 